Article

\title{
A Semi-Analytical Methodology for Multiwell Productivity Index of Well-Industry-Production-Scheme in Tight Oil Reservoirs
}

\author{
Guangfeng Liu ${ }^{1}\left(\mathbb{D}\right.$, , Zhan Meng ${ }^{1, *}$, Yan Cui ${ }^{2}$, Lu Wang ${ }^{1}$, Chenggang Liang ${ }^{3}$ and \\ Shenglai Yang ${ }^{1, *}$ \\ 1 CMOE Key Laboratory of Petroleum Engineering, China University of Petroleum-Beijing, Beijing 102249, \\ China; lgf@cup.edu.cn (C.L.); wlhmhxydh@163.com (L.W.) \\ 2 Changqing Oilfield Company, PetroChina, Xi'an 710021, China; cuiyan01_cq@petrochina.com.cn \\ 3 Xinjiang Oilfield Company, PetroChina, Kelamayi 831511, China; liangchenggang@petrochina.com.cn \\ * Correspondence: mengzhan1990@126.com (Z.M.); yangsl1961@126.com (S.Y.); Tel.: +86-010-8973-2192 (Z.M.)
}

Received: 22 March 2018; Accepted: 19 April 2018; Published: 25 April 2018

\begin{abstract}
Recently, the well-industry-production-scheme (WIPS) has attracted more and more attention to improve tight oil recovery. However, multi-well pressure interference (MWPI) induced by well-industry-production-scheme (WIPS) strongly challenges the traditional transient pressure analysis methods, which focus on single multi-fractured horizontal wells (SMFHWs) without MWPI. Therefore, a semi-analytical methodology for multiwell productivity index (MPI) was proposed to study well performance of WIPS scheme in tight reservoir. To facilitate methodology development, the conceptual models of tight formation and WIPS scheme were firstly described. Secondly, seepage models of tight reservoir and hydraulic fractures (HFs) were sequentially established and then dynamically coupled. Numerical simulation was utilized to validate our model. Finally, identification of flow regimes and sensitivity analysis were conducted. Our results showed that there was good agreement between our proposed model and numerical simulation; moreover, our approach also gave promising calculation speed over numerical simulation. Some expected flow regimes were significantly distorted due to WIPS. The slope of type curves which characterize the linear or bi-linear flow regime is bigger than 0.5 or 0.25 . The horizontal line which characterize radial flow regime is also bigger 0.5 . The smaller the oil rate, the more severely flow regimes were distorted. Well rate mainly determines the distortion of MPI curves, while fracture length, well spacing, fracture spacing mainly determine when the distortion of the MPI curves occurs. The bigger the well rate, the more severely the MPI curves are distorted. While as the well spacing decreases, fracture length increases, fracture spacing increases, occurrence of MWPI become earlier. Stress sensitivity coefficient mainly affects the MPI at the formation pseudo-radial flow stage, almost has no influence on the occurrence of MWPI. This work gains some addictive insights on multi-well performance for WIPS scheme in tight reservoir, which can provide considerable guidance on fracture properties estimation as well as well adjustment of production operation for WIPS scheme.
\end{abstract}

Keywords: tight oil reservoir; well-industry-production-scheme; multiwell productivity index; multiwell pressure interference; transient pressure analysis

\section{Introduction}

In recent years, with the increasing global energy demand, as unconventional oil resources, tight oil has become a new hot spot of unconventional oil and oil exploration and development after shale 
oil. Annual Energy Outlook released by EIA predicts that U.S. tight oil production will increase to more than 6 million barrels per day $(\mathrm{b} / \mathrm{d})$ in the coming decade, making up most of total U.S. oil production [1]. The production of tight oil has increased significantly since 2010, driven by horizontal well drilling and hydraulic fracturing technologies [2]. Therefore, it is very important to accurately predict the horizontal well productivity after fracturing for reservoir engineers.

A large number of productivity models of fracturing horizontal well have been established over the past decades. Based on Source/Green's function method and Laplace transformation, Ozkan and Raghavan presented a semi-analytical model for a fracturing horizontal well [3,4]. Then, with the same method, Guo and Evans described a new semi-analytical solution for predicting the performance of a fracturing horizontal well [5]. After that, Wan and Aziz presented a new semi-analytical model for horizontal wells with multiple hydraulic fractures [6]. In this model, the fractures could be rotated at any angle to a well, and the angle was studied. In order to comprehensively study the influence of the fracture details on productivity, a hybrid numerical-analytical model was established [7].

Until 2006, the conception of Stimulated Reservoir Volume (SRV) was firstly proposed by Mayerhofer et al. using micro-seismic monitoring techniques [8]. Since then, productivity models of fracturing horizontal well became more complex, which was different from conventional double wings symmetric fractures. Ozkan et al. used a tri-linear flow model to simulate fluid flow and production behaviors of fracturing horizontal wells in unconventional reservoirs [9,10]. After that many works have been done to improve the tri-linear flow model [11-14].

And then, the stress sensitivity effect was taken into account in tight reservoirs. Wang et al. presented a semi-analytical model for a fracturing horizontal well [15]. By using the principles of Darcy's flow in the fractures and by using Fick's law in the matrix. Based on conformal transformation, Deng et al. described a new solution for fracturing horizontal wells, which could predict production rate and confirm reasonable fracture parameters [16].

Subsequent studies found that each SRV was not connected for multi-stage hydraulic fracturing technology. To account for the heterogeneity of SRV inside and outside region, mainly the change of porosity and permeability in SRV region, five-linear flow model for multi-stage hydraulically fractured horizontal wells was establish in tight oil reservoirs [17]. F. Dongyan et al. presented a composite model of multiple fractured horizontal well with considering the size of stimulated reservoir volume (SRV) [18]. For the further analysis of the heterogeneity of internal SRV, C. Xiao et al. presented a new analytical model for predicting the performance of a fracturing horizontal well [19].

Well-industry-production-scheme (WIPS) has been one of the most promising technologies to economically improve tight oil recovery. Micro-seismic fracturing mapping shows hydraulic fractures extending between wells, gaining the existence of multi-well pressure interference (MWPI) [20]. The enhancement of the possibilities of MWPI in WIPS scheme severely increases the burden of performance analysis, this technology strongly challenges existing well performance analysis methods without pressure interference [21]. A semi-analytical methodology for multiwell productivity index is proposed to study well performance of WIPS scheme in tight reservoir.

Described below are the attributes of our methodology framework. Section 2 describes the conceptual model of WIPS and development of semi-analytical model of multiwell productivity index in detail. Section 3 systematically implemented model validation, methodology to identify the IWPI and sensitivity analysis in WIPS scheme. Finally, Section 4 summarizes our contribution and promising work in the future.

\section{Development of MPI Model for WIPS Scheme}

\subsection{Conceptual Model}

\subsubsection{WIPS Scheme}

Figure 1 illustrates the WIPS schemes in a tight oil reservoir. The whole oil field can consist of several independent WIPS scheme. Although every WIPS scheme also can interfere with each other 
when the wells produce enough long time, in this paper, we mainly focus on WIPS within one WIPS scheme at the early-intermediate production period. Each WIPS scheme contains several SMFHWs which produce using same platform. Figure $1 \mathrm{a}$, b illustrate the micro-seismic surveillance within one WIPS scheme and the corresponding idealized scenarios of hydraulic fractures.

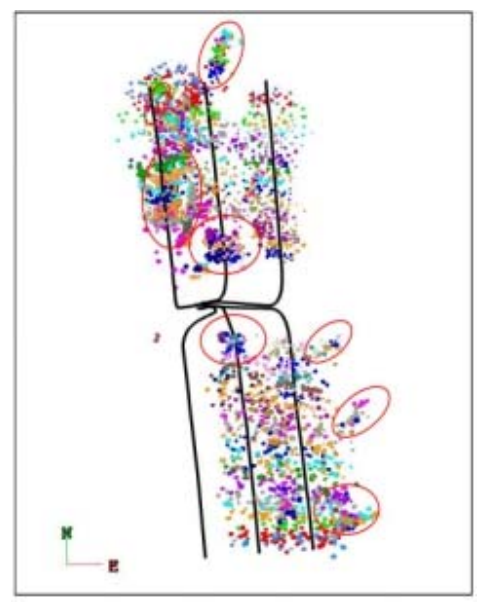

(a)

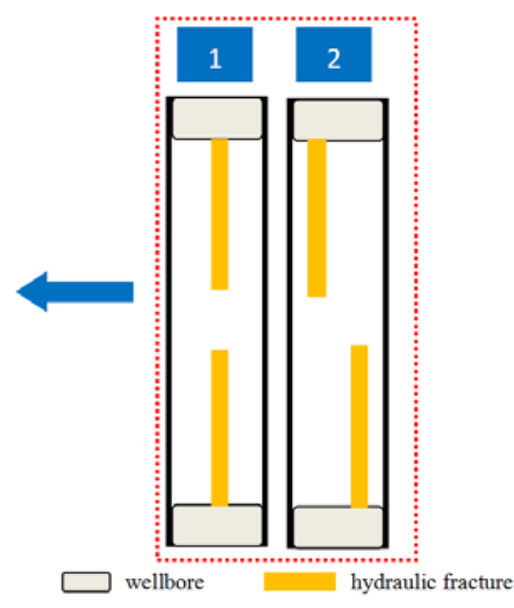

(b)

Figure 1. WIPS scheme in tight oil reservoir, (a) is one possible scenarios of WIPS scheme; (b) is idealizations of two possible illustration of hydraulic fractures.

\subsubsection{Tight Oil Formation Model}

Because the reservoir thickness is much smaller than its radius, the conceptual model of tight formation can be interpreted as a two-dimensional flat. Tight reservoirs are assumed to be isotropic reservoirs, including natural fractures (NFs) systems and matrix systems, and bounded by upper and lower impermeable formations.

In order to describe our method conveniently, we chose two SMFHWs for our research purpose. However, our approach can be easily extended to more than three SMFHWs WIPS scenarios. Two SMFHWs produced at constant oil rate $q_{1}$ and $q_{2}$. Fluid flows into HFs from tight oil reservoir and is assumed to $q_{f}$. In addition, there are some other assumptions as follows:

- hydraulic fractures in horizontal wells are distributed symmetrically and penetrate the reservoir completely;

- The thickness of reservoir is $h$, the initial pressure is $P i$ and the initial temperature is $T$;

- Fluid flow in NFs system and matrix satisfies Darcy's law. Natural fractures (NFs) system consider the effect of stress sensitivity;

- Considering the compressibility of fluid, assuming the compression coefficient is a constant value;

- Neglecting the influence of gravity and capillary force;

- Wellbore storage is considered.

\subsection{Mathematical Model}

\subsubsection{Parameter Description of HFs}

As shown in Figure 2, for well1, the fracture permeability is $k_{f 1}$, the fracture width is $w_{f 1}$, the fracture half-length is $L_{f 1}$. For well2, the fracture permeability is $k_{f 2}$, the fracture width is $w_{f 2}$, the fracture half-length is $L_{f 2}$. The distance between two well is $L_{w}$. the distance of two hydraulic fractures is $L_{f 12}$. To establish a mathematical model, we first subdivide the HFs systems of WIPS scheme. We assume that each hydraulic fracture of well1 is divided into $N_{1}$ sub-fracture segments, each hydraulic fracture of well 2 is divided into $N_{2}$ sub-fracture segments. The hydraulic fracture 
number of well1 is $M_{1}$. The hydraulic fracture number of well2 is $M_{2}$. The length of each fracture segment for well1 and well2 can be presented as $\Delta L_{f 1}, \Delta L_{f 2}$, respectively. We can summarize fracture properties as follows:

- Well 1: $N_{1}, M_{1}, L_{f 1}, \Delta L_{f 1}$

- Well 2: $N_{2}, M_{2}, L_{f 2}, \Delta L_{f 2}$

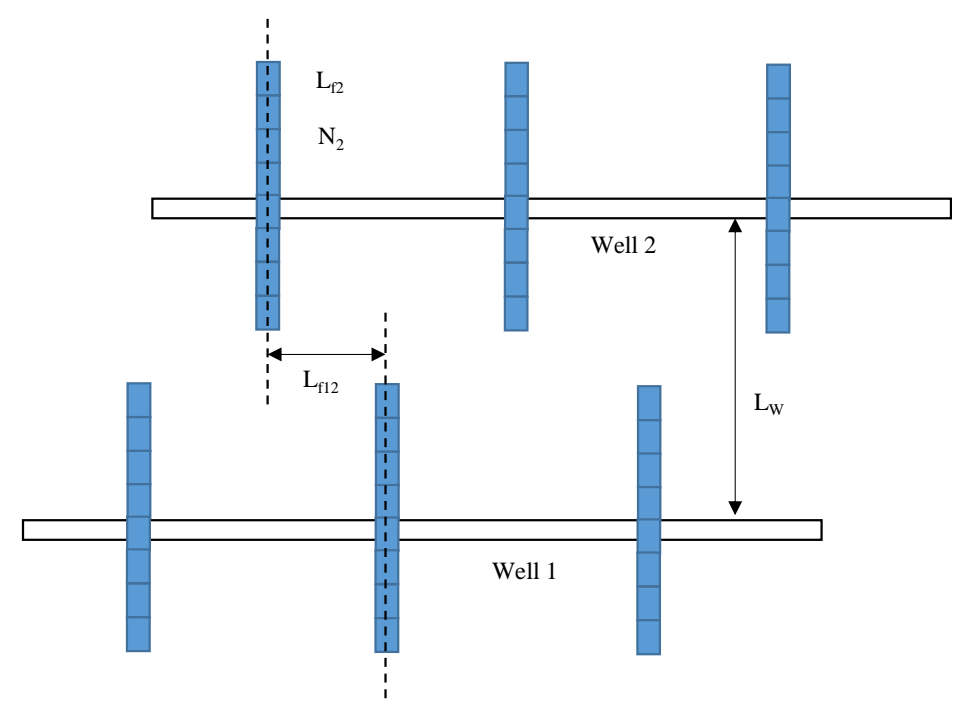

Figure 2. Subdivision of hydraulic fractures in WIPS scheme.

\subsubsection{Seepage Model in Tight Reservoir System}

We characterized tight reservoir as dual-porosity continuum and fracture system. Fluid flow in the fracture is considered compressible and can be described as a one-dimensional coordinate system (Figure 3). To obtain the solution in dual-porosity continuum, line-source function was a feasible approach [22,23]. After considering mass conservation principle, Darcy theory and motion equation, one can obtain the governing functions in dual-porosity continuum in Laplace domain as follows,

$$
\begin{gathered}
\frac{d^{2} \bar{\eta}}{d r_{D}^{2}}+\frac{1}{r_{D}} \frac{d \bar{\eta}}{d r_{D}}+\lambda\left(\overline{P_{m D}}-\bar{\eta}\right)=\omega S \bar{\eta} \\
-\lambda\left(\overline{P_{m D}}-\bar{\eta}\right)=(1-\omega) s \overline{P_{m D}}
\end{gathered}
$$

After substituting the fluid transferring from matrix into natural fracture system, the flow equations in the fractured system of is

$$
\frac{d^{2} \bar{\eta}}{d r_{D}^{2}}+\frac{1}{r_{D}} \frac{d \bar{\eta}}{d r_{D}}=f(s) \bar{\eta}
$$

where, $f(s)=\frac{(1-\omega) s \lambda}{(1-\omega) s+\lambda}+\omega s$.

The inner-boundary condition for a linear source can be as follows:

$$
\left.r_{D} \frac{d \bar{\eta}}{d r_{D}}\right|_{r_{D} \rightarrow 0}=-\overline{q_{D}}
$$

The outer-boundary condition for an infinite reservoir is

$$
\left.\bar{\eta}\right|_{r_{D} \rightarrow \infty}=0
$$


Based on the solution for a fully penetrating fracture is obtained [3,4], the general solution of Equation (3) can be given by

$$
\bar{\eta}\left(S, r_{D}\right)=A I_{0}\left(\sqrt{f(s)} r_{D}\right)+B K_{0}\left(\sqrt{f(s)} r_{D}\right)
$$

According to the boundary conditions, we have $A=0$ in Equation (4). Then, Equation (6) can be simplified

$$
\bar{\eta}\left(S, r_{D}\right)=\overline{q_{D}} K_{0}\left(\sqrt{f(s)} r_{D}\right)
$$

Using the principle of integration, pressure distribution at any random location $\left(x_{D}, y_{D}\right)$ can be obtained

$$
\bar{\eta}\left(S, x_{D}, y_{D}, x_{w D}, y_{w D}\right)=\frac{\overline{q_{D}}}{\Delta L_{i D}} \int_{x_{w D}-\Delta L_{i D} / 2}^{x_{w D}+\Delta L_{i D} / 2} K_{0}\left[\sqrt{f(s)} \sqrt{\left(x_{D}-u\right)+\left(y_{D}-y_{w D}\right)^{2}}\right] d u
$$

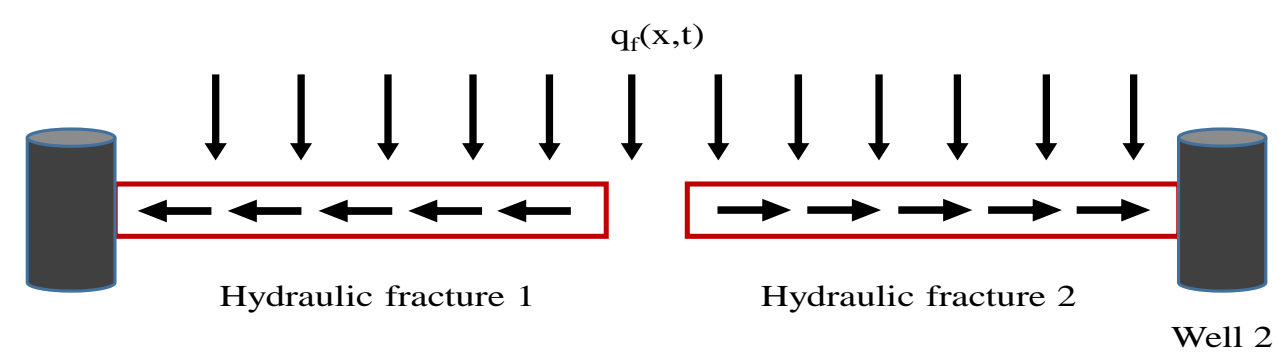

Well 1

Figure 3. Plan view of fluid flow within hydraulic fracture system.

\subsubsection{Seepage Model in Hydraulic Fracture System}

Semi-analytical method has been proposed to study the fluid flow within fracture system. Jia et al. [22], Zeng et al. [23] analyzed the unsteady state flow with considering fluid compressibility and rate accumulation, while Chen et al. [24] and Zhou et al. [25] analyzed the pseudo-steady state flow without considering fluid compressibility and rate accumulation. In our paper, we will establish a general model by considering the fluid compressibility and rate accumulation. When the fluid compressibility tends to be zero, the model can be equivalent to be a steady model.

Fluid flow in Hydraulic Fracture System can be presented as follows:

$$
\frac{\partial^{2} P_{f D}}{\partial l_{D}^{2}}-\frac{2 \pi q_{f D}\left(l_{D}\right)}{C_{f D}\left(l_{D}\right)}=\frac{1}{C_{\eta D}} \frac{\partial P_{f D}}{\partial t_{D}}
$$

And initial condition,

$$
P_{f D} \mid t_{D}=0=0
$$

Inner boundary contact with wellbore, thus

$$
\left.\frac{\partial P_{f D}}{\partial l_{D}}\right|_{l_{D}=0}=\frac{q_{c D}(0)}{C_{f D}}
$$

And there is no flow at the end of the fracture, so the outer boundary condition is

$$
\left.\frac{\partial P_{f D}}{\partial l_{D}}\right|_{l_{D}=L_{f D}}=0
$$




\subsection{Transient Pressure Analysis for WIPS Scheme}

\subsubsection{Tight Oil Reservoir System}

Traditionally, we used the superposition principle to calculate the pressure response of SMFHWs. Similarly, superposition principle was applied in our new model with WIPS scheme. Based on Equation (8), using the principle of integration, pressure distribution at the center of HFs system can be obtained:

$$
\begin{gathered}
\overline{P_{D}}\left(x_{D o}, y_{D o}\right)=\sum_{a=1}^{M_{1}} \sum_{b=j}^{N_{1}} \frac{\overline{q_{f i, j D}}}{\Delta L_{f 1 D}} \overline{P_{u D}}\left(x_{D 0}, y_{D 0}, x_{D a, b}, y_{D a, b}\right)+\sum_{i=1}^{M_{2}} \sum_{i=j}^{N_{2}} \frac{\overline{q_{f i, j D}}}{\Delta L_{f 2 D}} \overline{P_{u D}}\left(x_{D 0}, y_{D 0}, x_{D i, j}, y_{D i, j}\right) \\
o=1, \ldots,\left(M_{1} \times N_{1}+M_{2} \times N_{2}\right)
\end{gathered}
$$

where

$$
\begin{gathered}
\overline{P_{u D}}\left(x_{D 0}, y_{D 0}, x_{D a, b}, y_{D a, b}\right)=\int_{x_{D a, b}-\Delta L_{f 1 D} / 2}^{x_{D a, b}+\Delta L_{f 1 D} / 2} K_{0}\left[\sqrt{f(s)} \sqrt{\left(x_{D o}-u\right)+\left(y_{D o}-y_{D a, b}\right)^{2}}\right] d u \\
\overline{P_{u D}}\left(x_{D 0}, y_{D 0}, x_{D i, i}, y_{D i, i}\right)=\int_{x_{D i, i}-\Delta L_{f 2 D} / 2}^{x_{D i, i}+\Delta L_{f 2 D} / 2} K_{0}\left[\sqrt{f(s)} \sqrt{\left(x_{D o}-u\right)+\left(y_{D o}-y_{D i, j}\right)^{2}}\right] d u
\end{gathered}
$$

$\overline{P_{u D}}\left(x_{D 0}, y_{D 0}, x_{D a, b}, y_{D a, b}\right)$ is the pressure response due to the fluid flow in hydraulic fracture of horizontal well1; $\overline{P_{u D}}\left(x_{D 0}, y_{D 0}, x_{D i, i}, y_{D i, i}\right)$ is the pressure response due to the fluid flow in hydraulic fracture of horizontal well2.

\subsubsection{HFs System}

As shown in Figure 4, we can choose $i$-th fracture segment to analyze the flow equation in HFs System. Fluid in HFs System flow from $\varepsilon_{i 1}$ to $\varepsilon_{i 2}$. Hence, on the basis of Equation (9), the dimensionless governing function for this segment can be presented:

$$
\frac{d^{2} \overline{P_{F D}}}{d \varepsilon_{D}^{2}}-\frac{2 \pi}{C_{F D}} \cdot \overline{q_{c i D}}=\frac{u}{C_{\eta D}} \overline{P_{F D}}
$$

and boundary conditions at $\varepsilon_{i 1}$ and $\varepsilon_{i 2}$ given by

$$
\begin{aligned}
& \left.\frac{d \overline{P_{F D}}}{d \varepsilon_{D}}\right|_{\varepsilon_{D}=\varepsilon_{i 1 D}}=\frac{2 \pi}{C_{F D}} \overline{q_{c D}\left(\varepsilon_{i 1 D}\right)} \\
& \left.\frac{d \overline{P_{F D}}}{d \varepsilon_{D}}\right|_{\varepsilon_{D}=\varepsilon_{i 2 D}}=\frac{2 \pi}{C_{F D}} \overline{q_{c D}\left(\varepsilon_{i 2 D}\right)}
\end{aligned}
$$

Combining with Equations (16) to (18), the pressure distribution of the $i$-th fracture segment can be obtained. Zeng's method was applied to solve the fluid flow in the fracture segments [24]. The fluid flow equation of $i$-th fracture segment can be obtained by Laplace transformation.

$$
\overline{P_{i D}}\left(\varepsilon_{D}, u\right)=b_{i}\left(\varepsilon_{D}\right) \overline{q_{c i 2 D}}+c_{i}\left(\varepsilon_{D}\right) \overline{q_{c i 1 D}}+d_{i} \overline{q_{F i D}}
$$

Therefore, pressure of $i$-th fracture segment can be obtained

$$
\overline{P_{i c D}}\left(\frac{\varepsilon_{i 1 D}+\varepsilon_{i 2 D}}{2}, u\right)=b_{i}\left(\frac{\varepsilon_{i 1 D}+\varepsilon_{i 2 D}}{2}\right) \overline{q_{c i 2 D}}+c_{i}\left(\frac{\varepsilon_{i 1 D}+\varepsilon_{i 2 D}}{2}\right) \overline{q_{c i 1 D}}+d_{i} \overline{q_{F i D}}
$$

where

$$
b_{i}\left(\varepsilon_{D}\right)=\frac{2 \pi}{C_{F D} \sqrt{\frac{u}{C_{\eta D}}}}\left\{\frac{2 \cosh \left[\left(\varepsilon_{D}-\varepsilon_{i 1 D}\right) \sqrt{\frac{u}{C_{\eta D}}}\right]}{e^{2\left(\varepsilon_{i 2 D}-\varepsilon_{i 1 D}\right)} \sqrt{\frac{u}{C_{\eta D}}}-1}\right\}+e^{-\left(\varepsilon_{D}-\varepsilon_{i 1 D}\right) \sqrt{\frac{u}{C_{\eta D}}}}
$$




$$
\begin{gathered}
c_{i}\left(\varepsilon_{D}\right)=\frac{-2 \pi}{C_{F D} \sqrt{\frac{u}{C_{\eta D}}}}\left\{\frac{2 \cosh \left[\left(\varepsilon_{i 2 D}-\varepsilon_{D}\right) \sqrt{\frac{u}{C_{\eta D}}}\right]}{e^{2\left(\varepsilon_{i 2 D}-\varepsilon_{i 1 D}\right)} \sqrt{\frac{u}{C_{\eta D}}}-1}\right\}+e^{-\left(\varepsilon_{i 2 D}-\varepsilon_{D}\right) \sqrt{\frac{u}{C_{\eta D}}}} \\
d_{i}\left(\varepsilon_{D}\right)=\frac{-2 \pi C_{\eta D}}{C_{F D} u}
\end{gathered}
$$

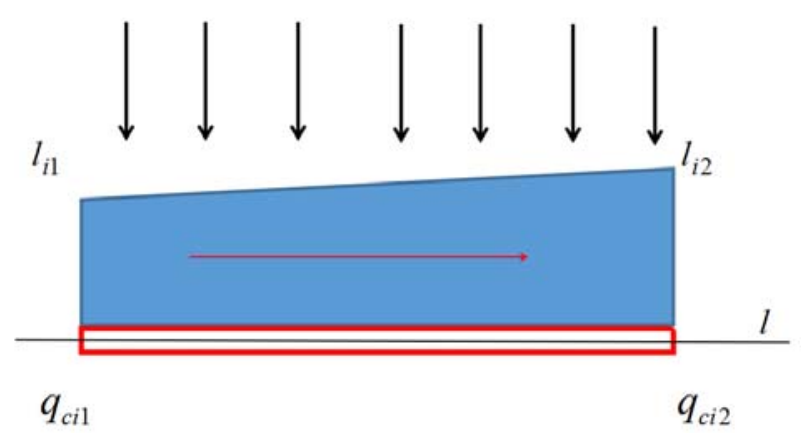

Figure 4. Illustration of fluid flow within $i$-th fracture segment.

\subsubsection{Solution Methodology}

Observation from the proposed equations, there are three unknowns for each fracture segment: $\overline{P_{f D i 1}}, \overline{q_{c D i 1}}, \overline{q_{f D i 1}}$. Then, the total number of unknowns are equal to $\left[3\left(M_{1} \times N_{1}+M_{2} \times N_{2}\right)+2\right]$. We can obtain a closed [3 $\left.\left(M_{1} \times N_{1}+M_{2} \times N_{2}\right)+2\right]$-order matrix from the following conditions:

For each fracture segment, the pressure obtained from reservoir fluid flow calculation and fluid flow calculation should be equal.

$$
\overline{P_{D}}\left(x_{D i}, y_{D i}\right)=\overline{P_{i c D}}
$$

Equation (24) applies to every fracture segment, so we can obtain more $\left(M_{1} \times N_{1}+M_{2} \times\right.$ $\mathrm{N}_{2}$ )-equations.

Besides, the fluid rate and pressure at adjacent joints between two fracture segments should be same, namely,

$$
\begin{gathered}
q_{c i 2 D}=q_{c(i+1) 1 D} \\
b_{i}\left(\varepsilon_{i 2 D}\right) \overline{q_{c i 2 D}}+c_{i}\left(\varepsilon_{i 2 D}\right) \overline{q_{c i 1 D}}+d_{i} \overline{q_{F i D}}=\overline{P_{(i+1) D}}\left(\varepsilon_{(i+1) 1 D}, u\right)
\end{gathered}
$$

Another two equations are required to form a closed matrix. Well1 and well2 is produced at constant rate $q_{1}$ and $q_{2}$, respectively. Here, we define a new variable $\alpha$, represents the ratio between $q_{1}$ and $\left(q_{1}+q_{2}\right)$, namely, $\varepsilon=q_{1} /\left(q_{1}+q_{2}\right)$. Therefore,

$$
\begin{gathered}
\sum_{i=1}^{M_{1}} \overline{q_{c D i(0)}}=\frac{\varepsilon}{u} \\
\sum_{i=1}^{M_{2}} \overline{q_{c D 2(0)}}=\frac{1-\varepsilon}{u}
\end{gathered}
$$

Finally, we can obtain a closed [ $\left.3\left(M_{1} \times N_{1}+M_{2} \times N_{2}\right)+2\right]$-order matrix. Gauss elimination and Stehfest numerical algorithm [26] can be used to obtain pressure distribution solutions for the new model with WIPs scheme. Because of the solution in Laplace domain, the wellbore storage and skin factor can be easily added into the solution according to Duhamel's theorem. Therefore, the solution can be presented as follows:

$$
\bar{\eta}_{D w f i n 1}=\frac{\bar{\eta}_{D w f 1} / \varepsilon}{1+u^{2} C_{D 1} \bar{\eta}_{D w f 1} / \varepsilon}
$$




$$
\bar{\eta}_{D w f i n 2}=\frac{\bar{\eta}_{D w f 2} /(1-\varepsilon)}{1+u^{2} C_{D 2} \bar{\eta}_{D w f 2} /(1-\varepsilon)}
$$

where, $C_{D 1}, C_{D 1}$ are dimensionless wellbore storage coefficient for well1 and well 2 which are defined as $C_{D 1}=\frac{C_{1}}{2 \pi L_{r e f}^{2} h \phi C_{t}}, C_{D 2}=\frac{C_{2}}{2 \pi L_{r e f}^{2} h \phi C_{t}}$.

The stress sensitivity of NFs also can be taken into consideration. The bottom hole pressure in real space can be easily obtained Using the Stehfest numerical invention algorithm [26].

$$
\begin{aligned}
& P_{W f D 1}=-\frac{1}{\zeta_{D}} \ln \left(1-\zeta_{D} \eta_{D w f i n 1}\right) \\
& P_{W f D 2}=-\frac{1}{\zeta_{D}} \ln \left(1-\zeta_{D} \eta_{D w f i n 2}\right)
\end{aligned}
$$

To analyze well performance in WIPS, Dimensionless multiwell productivity index $J_{1 D}$ and $J_{2 D}$, can be given as,

$$
J_{1 D}=\frac{B \mu q_{1} /\left(P_{1}-P_{2}\right)}{2 \pi K_{r i} h}=\frac{1}{P_{w f D 1}}, \quad J_{2 D}=\frac{B \mu q_{2} /\left(P_{1}-P_{2}\right)}{2 \pi K_{r i} h}=\frac{1}{P_{w f D_{2}}}
$$

\section{Results and Discussion}

In this section, three cases are studied, (I) model verification, (II) identification of flow regimes, and (III) sensitivity analysis. Case I is used for model verification using numerical simulation; Case II is applied to identify flow regimes based on special pressure-transient characteristics; Case III is

\begin{tabular}{|c|c|c|}
\hline Type & Parameters & Value \\
\hline \multirow{7}{*}{ Reservoir } & Initial reservoir pressure, $P_{\mathrm{i}}(\mathrm{MPa})$ & 25 \\
\hline & Formation temperature, $T(\mathrm{~K})$ & 330 \\
\hline & Formation thickness, $h(\mathrm{~m})$ & 10 \\
\hline & Total compressibility of reservoir, $C_{t}\left(\mathrm{MPa}^{-1}\right)$ & $2.5 \times 10^{-4}$ \\
\hline & Porosity of reservoir $\Phi$ (fraction) & 0.06 \\
\hline & Reservoir area, $(\mathrm{m} \times \mathrm{m})$ & $1000 \times 500$ \\
\hline & Initial natural fracture permeability, $k_{r i}(\mathrm{D})$ & 0.001 \\
\hline \multirow{7}{*}{ Well1 } & Hydraulic fracture permeability, $k_{f 1}(\mathrm{D})$ & 10 \\
\hline & Hydraulic-fracture width, $w_{f 1}(\mathrm{~m})$ & 0.005 \\
\hline & Hydraulic-fracture half-length, $L_{f 1}(\mathrm{~m})$ & 30 \\
\hline & Hydraulic-fracture number, $M_{1}$ & 4 \\
\hline & Total compressibility of hydraulic fracture, $C_{t f 1}\left(\mathrm{MPa}^{-1}\right)$ & $3.5 \times 10^{-4}$ \\
\hline & Hydraulic-fracture porosity, $\Phi_{f 1}$ (fraction) & 0.35 \\
\hline & Wellbore length, $L_{w 1}(\mathrm{~m})$ & 1000 \\
\hline \multirow{7}{*}{ Well2 } & Hydraulic fracture permeability, $k_{f 2}(\mathrm{D})$ & 10 \\
\hline & Hydraulic-fracture width, $w_{f 2}(\mathrm{~m})$ & 0.005 \\
\hline & Hydraulic-fracture half-length, $L_{f 2}(\mathrm{~m})$ & 30 \\
\hline & Hydraulic-fracture number, $M_{2}$ & 4 \\
\hline & Total compressibility of hydraulic fracture, $C_{t f 2}\left(\mathrm{MPa}^{-1}\right)$ & $3.5 \times 10^{-4}$ \\
\hline & Hydraulic-fracture porosity, $\Phi_{f 2}$ (fraction) & 0.35 \\
\hline & Wellbore length, $L_{w 2}(\mathrm{~m})$ & 1000 \\
\hline
\end{tabular}
sensitivity analysis of key parameters on pressure-transient response of WIPS scheme. The relevant parameters are shown in Table 1.

Table 1. The basic input parameters in numerical simulation.

\subsection{Model Validation}

At present, there is no analytical or semi-analytic model for multi-well interference. Therefore, we choose commercial software to verify the accuracy of our model, CMG-2010 (Computer Management Group Ltd., Calgary, AB, Canada), and the top view of WIPS scheme is shown in Figure 5. 
The numerical simulation model consists of two horizontal wells. Each horizontal well contains four hydraulic fractures with a fracture spacing of $250 \mathrm{~m}$. Double klinkenberg permeability- logarithmic spacing-local grid refinement (DK-LS-LGR) technology was used to characterize hydraulic fractures system. To avoid the same pressure type curve between the two horizontal wells, the oil ratio was assumed to 1:4. As shown in Figure 6, we can get a good fit between the numerical simulation and the theoretical model, which shows that the new model is reliable.
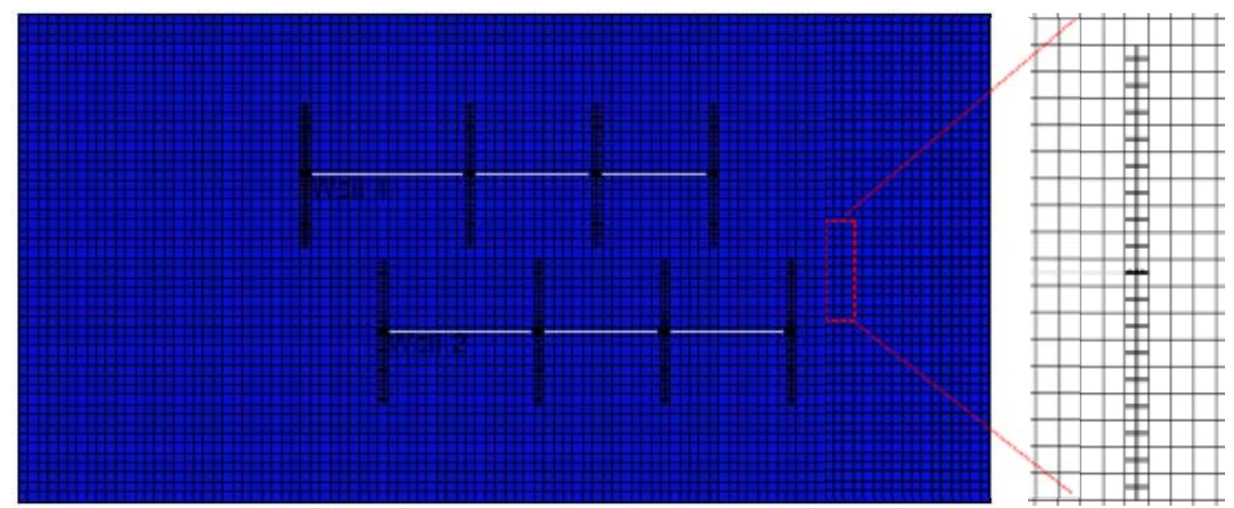

Figure 5. Top view of the numerical model of Case II in CMG module.

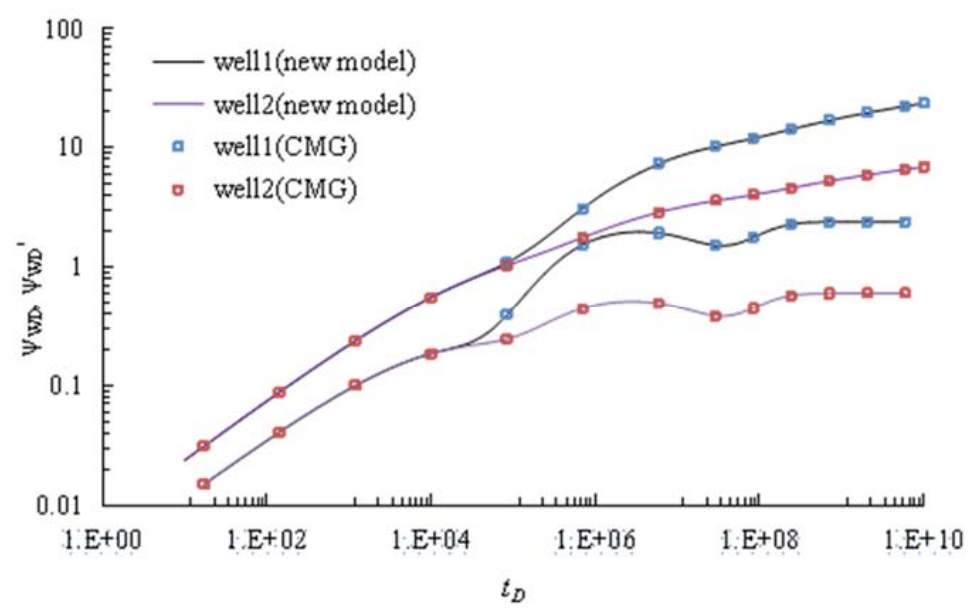

Figure 6. Comparison of our results of model with that of CMG simulator.

On the premise of ensuring the accuracy of the model, we tested the calculation speed of the model. Under the same hardware condition, the running time of the semi-analytical model and the numerical simulation model were 245.2 and $273.5 \mathrm{~s}$, respectively.

\subsection{Identification of MWPI Using Flow Regime}

In this section, we will mainly focus on identifying the MWPI phenomenon based on the special characteristic of pressure response curves. Common parameters were set for those two wells: $M_{1}=3$, $M_{2}=3, \zeta_{D}=0.05, \lambda=0.002, \gamma=0.15, \omega=0.0035, C_{\eta D}=10^{5}$. Setting consistent fracture properties (conductivity and half-length) $L_{f 1 D}=L_{f D 2}=200, C_{f 1 D}=C_{f 2 D}=10$. Another two parameters, $L_{w D}$ and $L_{f 12 D}$, can be mathematically described as follows:

- $\quad$ Figure $1 b(1): L_{w D}=L_{f 1 D}+L_{f D 2}+100=500$, and $L_{f 12 D}=0$;

- $\quad$ Figure $1 b(2): L_{w D}=L_{f 1 D}+L_{f D 2}+100=500$, and $L_{f 12 D}=500$.

Dimensionless pressure (DP) and the dimensionless pressure derivative (DPD) of WIPS scheme are shown in Figure 7. To clearly describe the type curves, we will compare the characteristics of 
pressure curves between WIPS and SMFHWs. By Comparing between SMFHWs and WIPS scheme, we can add some additional information and better explanation into these distorted flow regimes, which can be described in detail as follows:

Regime I: The pure wellbore storage period regime. DP curve and DPD curve align, and the slope of curves are equal to 1 . This stage is mainly controlled by wellbore storage effect and difficult to be impacted by the MWPI. Thus, the type curve of WIPS and SMFHWs overlap with each other.

Regime II: The transition flow regime. The early stage of this regime gradually derives from the straight line which has unit slope. This stage is mainly controlled by fluid properties and also difficult to be impacted by MWPI. Thus, the type curve of WIPS and SMFHWs also overlap with each other.

Regime III: The linear flow regime within HFs. This stage is mainly dominated by fracture conductivity. At this linear flow regime, and also difficult to be impacted by MWPI. Thus, the type curve of WIPS and SMFHWs also overlap with each other.

Regime IV: The bi-linear flow regime. This stage is mainly controlled by fracture length. At this bi-linear flow regime, we can start to detect the MWPI for type Figure 1b (1). The slope of the DPD curves is actually bigger than 0.25 . The distortion degree of pressure curve for small oil rate is also more significant than that of big oil rate. However, it is still difficult to be impacted by the MWPI for type Figure $1 b(2)$, Thus, the type curve of WIPS and SMFHWs still overlap with each other.

Regime V: The early pseudo-radial flow regime. This stage is mainly dominated by fracture spacing. At this pseudo-radial flow regime, we can start to detect the MWPI for type Figure $1 \mathrm{~b}$ (2). The slope of the DPD curves is actually bigger than 0 . The distortion degree of pressure curve for small oil rate is more significant that that of big oil rate.

Regime VI: The intermediate-time linear flow regime. This stage is mainly dominated by wellbore length. At this intermediate-time linear flow regime, we also can detect the MWPI for type Figure $1 \mathrm{~b}$ (1) and Figure $1 b$ (2). The slope of the DPD curves is actually bigger than 0.5. The distortion degree of pressure curve for small oil rate is more significant that that of big oil rate.

Regime VII: The pseudo-steady diffusion regime between the matrix and natural fractures. The main feature of type curves is marked by a "dip" shape in DPD curves.

Regime VIII: The late-time pseudo-radial flow regime. The shape of DPD curve is a horizontal line. The value of this horizontal well is equal to 0.5 .

This section can clearly demonstrate the existence of multi-well pressure interference in WIPS by using the transient-pressure analysis. Furthermore, to analyze the well performance in WIPS with the influence of multi-well pressure interference, Figure 8 illustrates the dimensionless multi-well productivity index. Comparing to the SMFHWs, the well performance severely decreases. Figure 8 also shows that the different location of fractures leads to different occurrence periods of multi-well pressure interference.

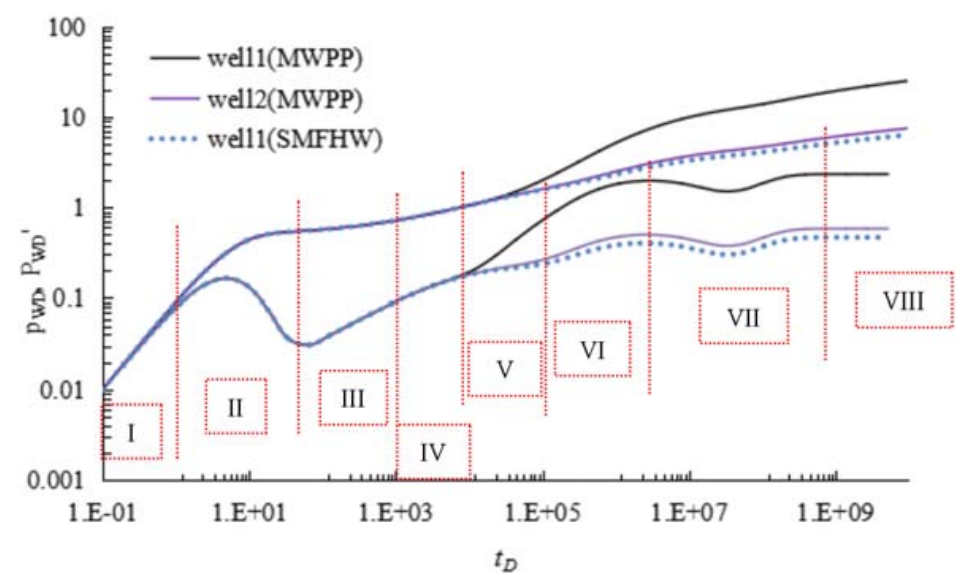

(a)

Figure 7. Cont. 


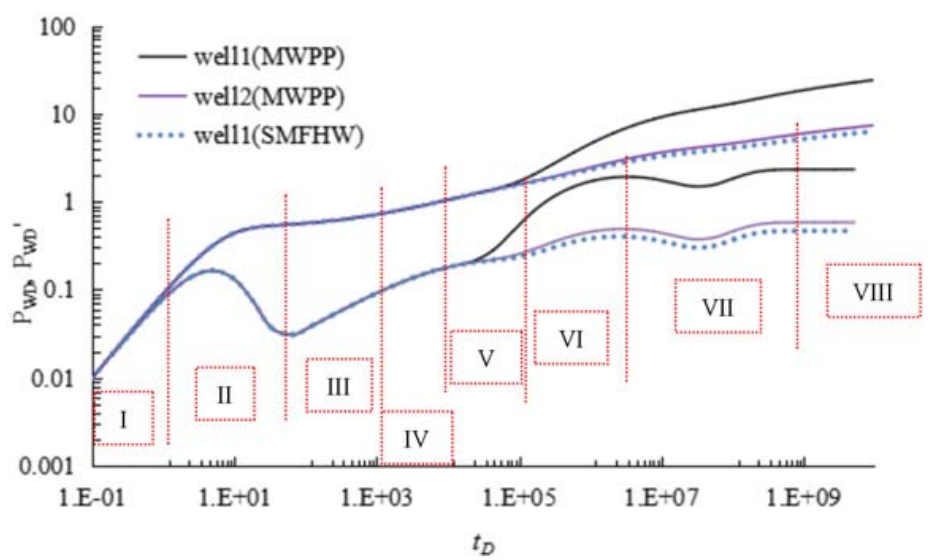

(b)

Figure 7. Comparison of pressure curves between WIPS and SMFHWs $\left(q_{1}: q_{2}=1: 4\right)$ : (a) Figure $1 \mathrm{~b}$, case 1 ; (b) Figure $1 b$, case 2.

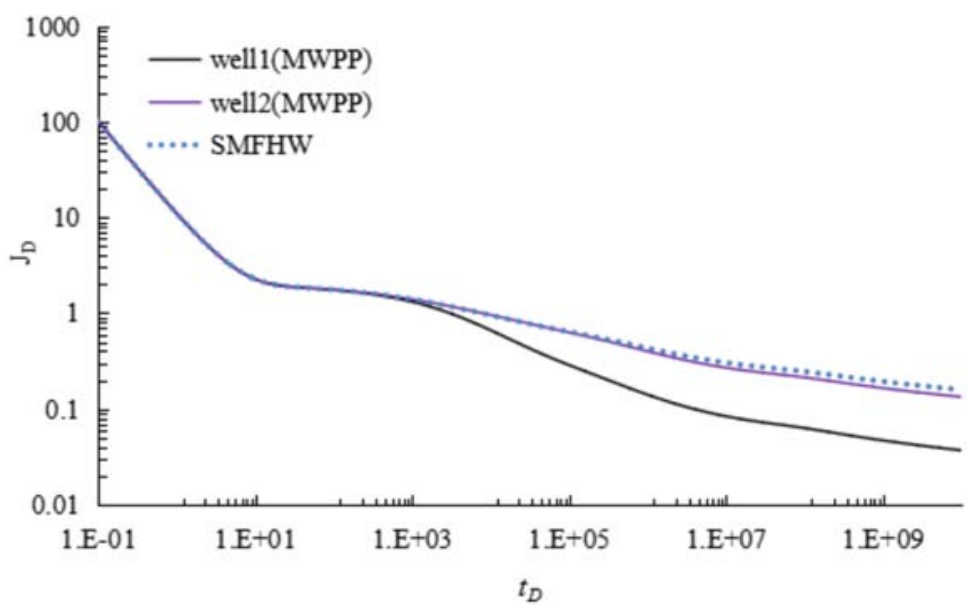

(a)

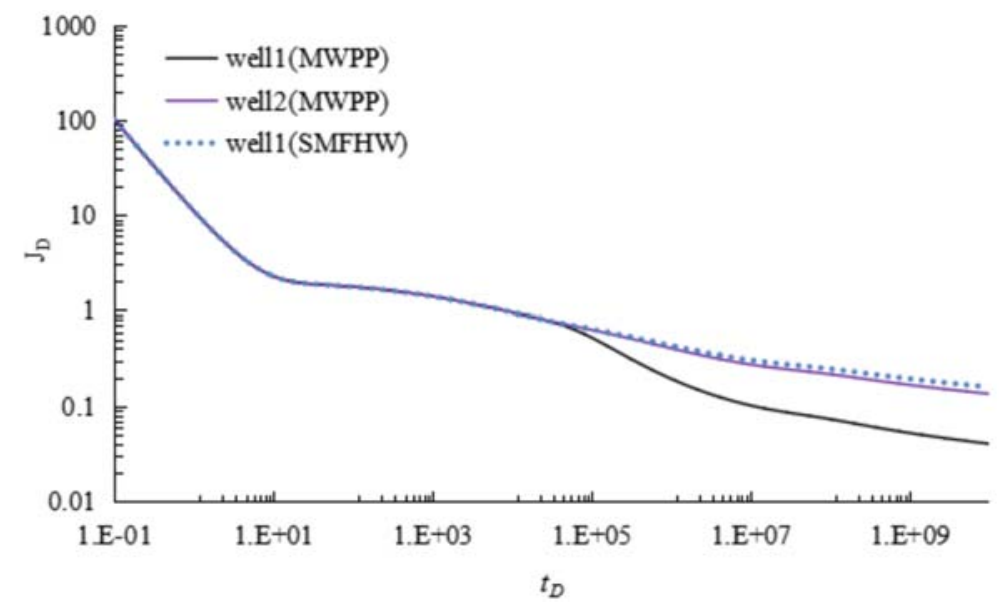

(b)

Figure 8. Comparison of productivity Index curves between WIPS and SMFHWs $\left(q_{1}: q_{2}=1: 4\right)$ : (a) Figure 1b, case 1; (b) Figure 1b, case 2. 


\subsection{Sensitivity Analysis}

In this section, we conduct some sensitivity analysis on MPI for WIPS scheme. The key factors that influence MPI for WIPS scheme include hydraulic fracture half-length $L_{f D}$, hydraulic fracture conductivity $C_{f D}$, hydraulic fracture spacing $L_{f 12 D}$, well spacing $L_{w D}$, ratio of well rate $\varepsilon$, stress sensitivity $\zeta_{D}$. Some dimensionless parameters can be: $S=0.2, C_{D}=10, \zeta_{\mathrm{D}}=0.05, \lambda=0.002, \gamma=0.15$, $\omega=0.0035, C_{\eta D}=105, L_{f 1 D}=2000, L_{f 2 D}=2000, L_{w D}=3000, L_{f 12 D}=1000 . C_{f 1 D}=50, C_{f 1 D}=50$. The results are discussed in detail as follows:

\subsubsection{Ratio of Well Rate, $\varepsilon$}

We set $\varepsilon$ to be $0.1,0.3,0.5$ respectively. Figure 9 shows effects of ration of well rate on pressure performance for WIPS scheme. We can judge the occurrence of MWPI by whether the MPI curves of two wells overlap together. The smaller the well rate, the more severely the curves are distorted. Moreover, the smaller the well rate, the smaller the dimensionless multi-well productivity index. Therefore, we can judge the well rate of two wells based on the relative position of the MPI curves. We also can summarize that the ratio of well rate $\varepsilon$ approximately has no any influence on the time when the MWPI occurs.

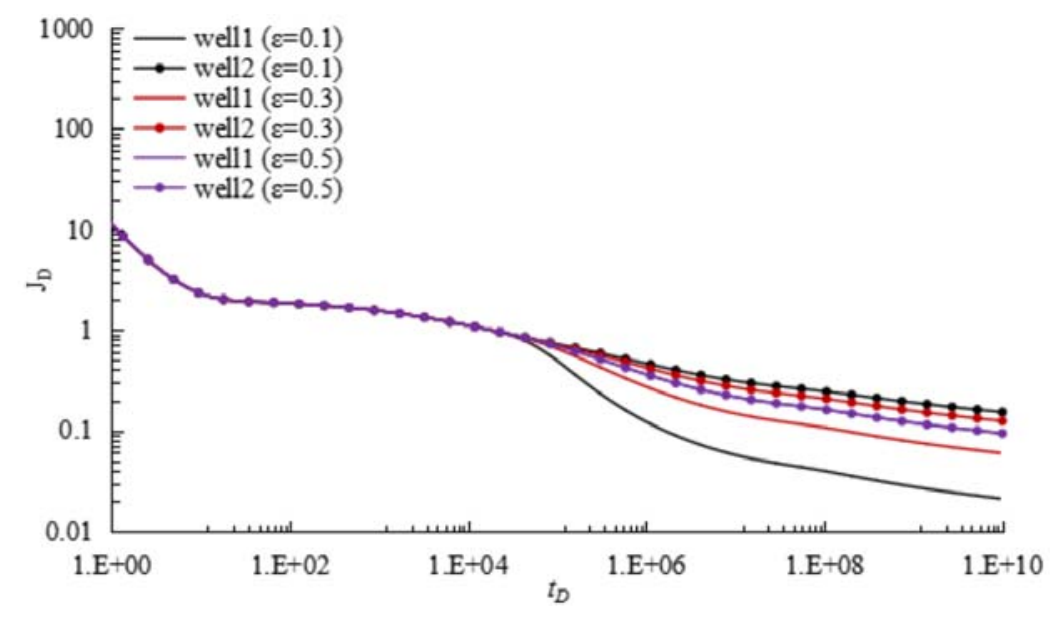

Figure 9. Effects of ratio of oil rate on MPI for WIPS scheme.

\subsubsection{Well Spacing, $L_{w D}$}

We set $L_{w D}$ to be $450,650,900$ respectively, and we also set $\varepsilon$ to be 1:4. Figure 10 illustrates the impacts of well spacing $L_{\mathrm{wD}}$ on pressure performance for WIPS scheme. Similarly, we also can judge the occurrence of MWPI by whether MPI curves of two wells overlap together. On condition of different well spacing $L_{w D}$, MWPI basically starts form first radial-flow regimes. As the well spacing $L_{w D}$ increases, the occurrence of MWPI becomes later, and thus MPI inversely increases. Besides, MPI curves will be split, the pressure curves will overlap again subsequently. We also can clearly observe another phenomenon that well spacing almost does not distort the shape of MPI curves, the MPI curves just move upward or downward (the slope of MPI curves keeps constant). When the oil rate is big enough, the impacts of well spacing on multi-well interference can hardly be identified (the dot line). 


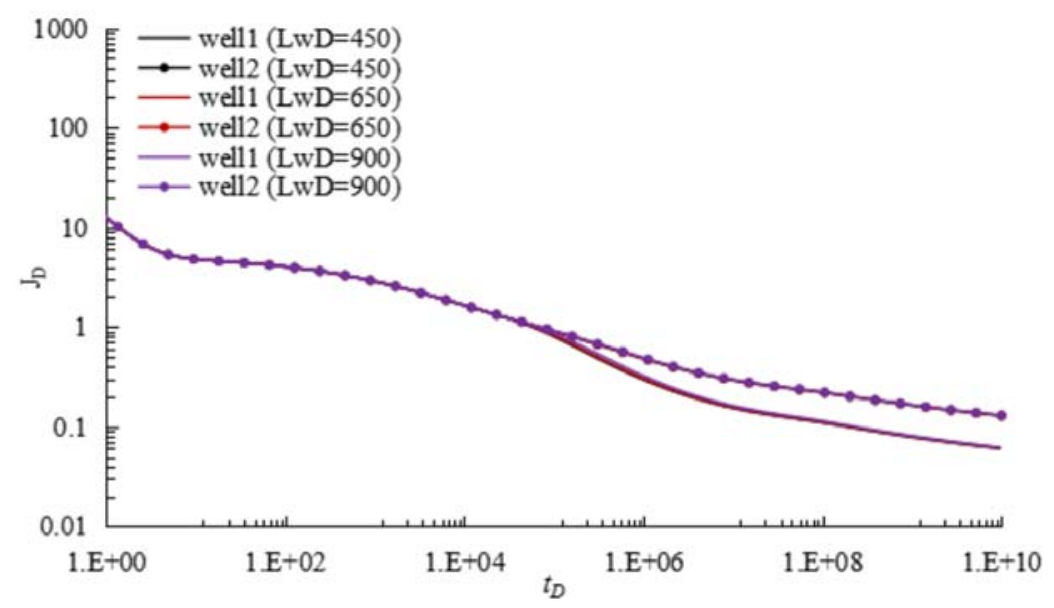

Figure 10. Effects of well spacing on MPI for WIPS scheme.

\subsubsection{Hydraulic Fracture Spacing, $L_{f 12 D}$}

We set $L_{f 12 D}$ to be $100,500,1000$ respectively, and we also set $\varepsilon$ to be $1: 4$. Figure 11 illustrates the impacts of hydraulic fracture spacing $L_{f 12 D}$ on pressure performance for WIPS scheme. Similar to the effects of well spacing on pressure curves, hydraulic fracture spacing mainly impacts the occurrence of MWPI and have no any influence on the distortion of flow regimes, the MPI curves just move upward or downward (the slope of MPI curves keeps constant). Different from the effects of well spacing, the impacts of fracture spacing is more significant than that of well spacing. When $L_{f 12 D}=100$, MWPI basically starts from first linear-flow regime. When $L_{f 12 D}=1000$, MWPI basically starts form first radial-flow regimes. As the fracture spacing $L_{f 12 D}$ increases, the occurrence of MWPI becomes later and thus MPI inversely increases. Similarly, when the oil rate is big enough, the impacts of fracture spacing on well performance also cannot be observed from MPI curves.

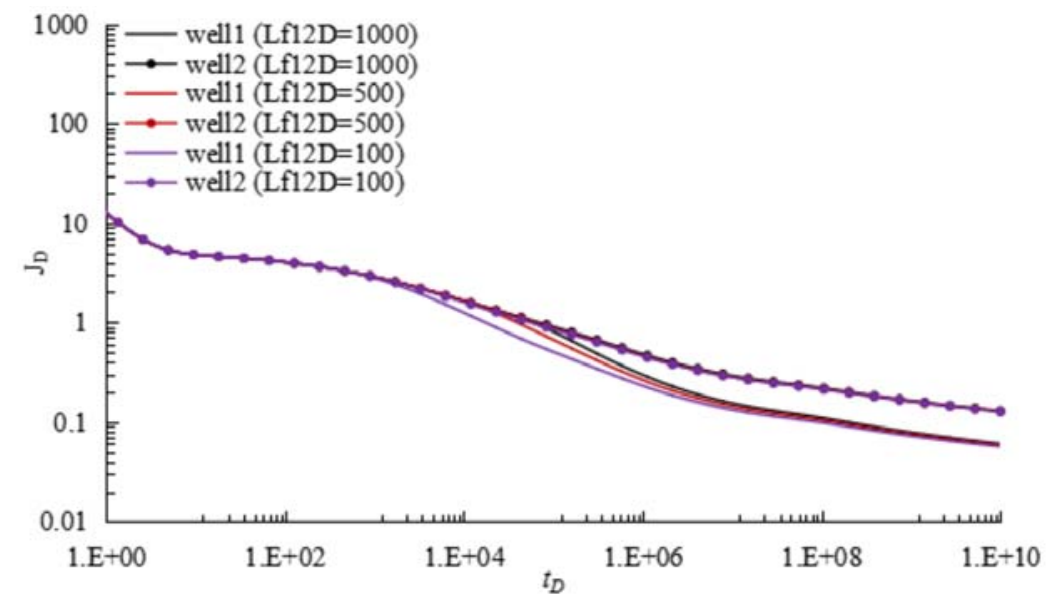

Figure 11. Effects of fracture spacing on MPI for WIPS scheme.

\subsubsection{Hydraulic Fracture Length, $L_{f 1 D}, L_{f 2 D}$}

We set $L_{f 1 D}=L_{f 2 D}=L_{f D}$ to be 1000,1500, 2000 respectively and $\varepsilon$ to be 1:4. Figure 12 illustrates the impacts of hydraulic fracture length $L_{f D}$ on pressure performance for WIPS scheme. We can systematically analyze the impacts of $L_{f D}$ on pressure performance from three aspects: (1) Before the occurrence of MWPI, for a certain fracture length, the pressure curves of two wells will overlap together. However, when fracture length is varying, the pressure curves will paralleled move upward or downward. As the fracture length increases, the pressure curves will paralleled move downward; 
(2) When the MWPI occurs, as the fracture length $L_{f D}$ increases, the occurrence of MWPI becomes earlier. For example, when $L_{f D}=2000$, MWPI basically starts form first linear-flow regime. When $L_{f D}=1000$, MWPI basically starts form first bi-linear-flow regime; (3) When MWPI reaches certain degree, the pressure curves will overlap again subsequently. The bigger the oil rate, the more lately the pressure curves overlap. For example, for well1, the pressure curves will overlap at second linear-flow regime, for well2, the pressure curves will overlap at pseudo-steady diffusion regime. In conclusion, fracture length impacts the whole flow regimes for WIPS scheme.

\subsubsection{Stress Sensitivity Coefficient, $\zeta$}

We set $\zeta_{D}$ to be $0,0.03,0.05$ respectively and $\varepsilon$ to be $1: 4$. Figure 13 illustrates the impacts of stress sensitivity coefficient $\zeta_{D}$ on pressure performance for WIPS scheme. It is hardly to find that stress sensitivity coefficient has some influences on the occurrence of MWPI. However, stress sensitivity coefficient can distort flow regimes at an inverse direction. For example, when $\zeta=0.03$, radial-flow regimes and pseudo-steady diffusion regimes are distorted. When $\zeta=0.05$, radial-flow regimes, pseudo-steady diffusion regimes and second linear-flow regime are distorted. As the $\zeta$ increases, the distortion of pressure curves becomes severe, and more flow regimes will be distorted.

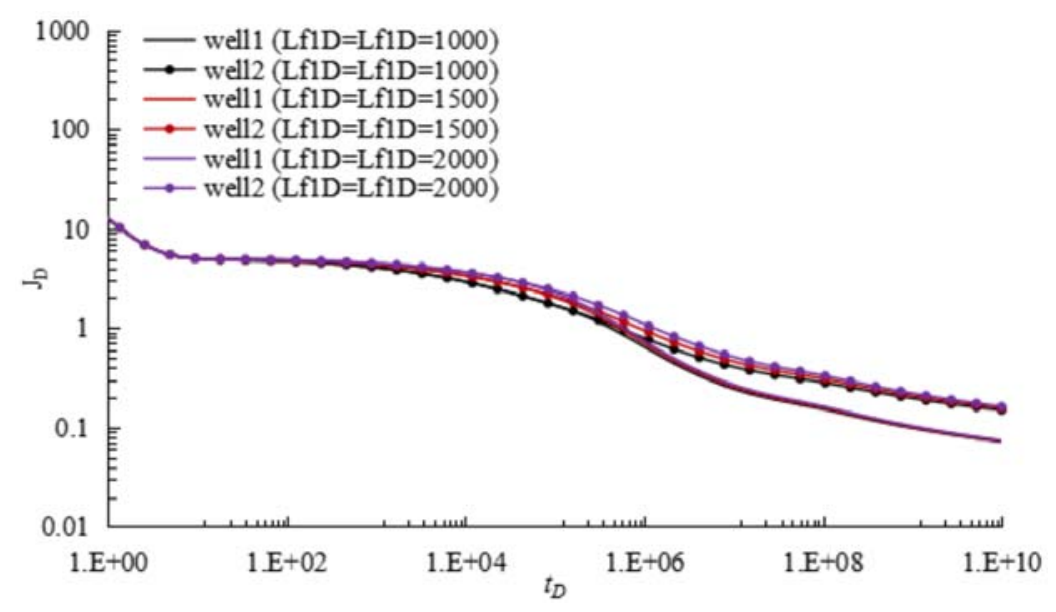

Figure 12. Effects of fracture half-length on MPI for WIPS scheme.

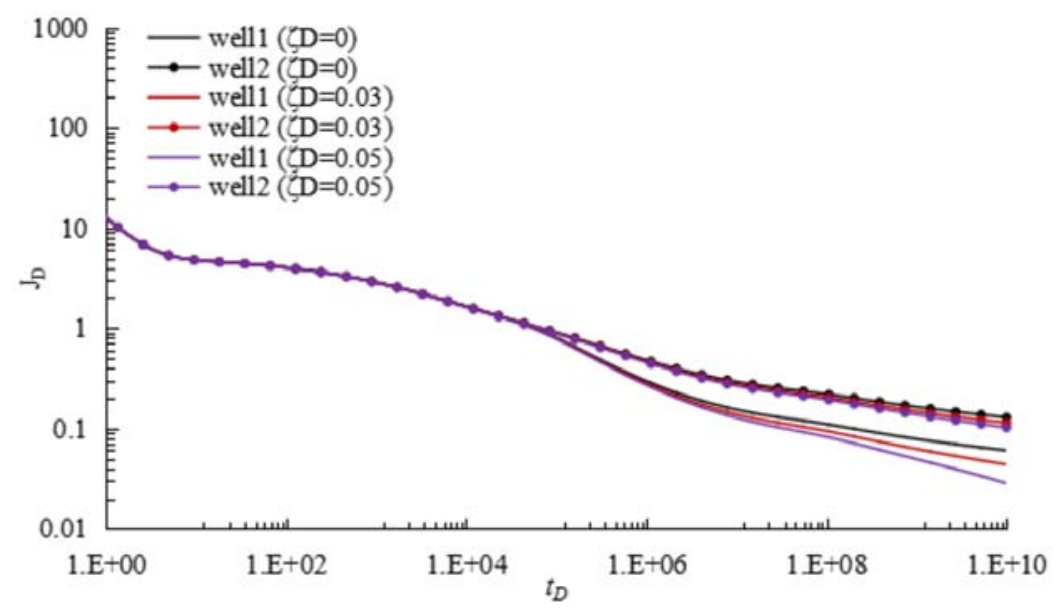

Figure 13. Effects of stress sensitivity coefficient on MPI for WIPS scheme. 


\subsection{Case Application}

In this section, we try to apply our methodology to a realistic case, Xinjiang Changji Oilfield is located in the east of the Zhungeer Basin, with an area of $1278 \mathrm{~km}^{2}$ and $150 \mathrm{~km}$ away from Urumqi, administrative district belongs to the Jimsar County of Xinjiang Uygur Autonomous Region. According to drilling data, the formation thickness of the area is $28-75 \mathrm{~m}$, an average of $53.5 \mathrm{~m}$, The average porosity of the reservoir is $10.99 \%$ and the permeability is $0.012 \mathrm{mD}$, which belong to the typical tight oil reservoir. To improve the oil recovery, well-industry-production-scheme (WIPS) is used, the illustration of the WIPS can be found in Figure 14. Because of some policy limitation, more detailed information cannot be specified. Our target wells are located at the north-west corner. This WIPS has 6 horizontal wells. Some fracturing parameters can be found in Table 2. Figure 15 shows the production scheme of the well JHW015, the production profile of other wells do not show here.

Given the specific parameters, including fracture length, number of fractures, well length, formation permeability, we estimate the well distance approximately using our proposed semi-analytical method. Here, we take the well JHW015 as an example to explain how to use our method. To begin with, the productivity index can be calculated using the historical production data, as Figure 15. Second, given some known parameters, several dimensionless productivity index curves can be plotted based on different well distance. Finally, we can match those curves to approximately estimate the possible well distance in this well-industry-production-scheme (WIPS). As one can see in Figure 16, at the early production stage, this is the fracturing fluid drawback from the formation with two-phase flow, as result, our model cannot match with this data, thus, we should avoid use the production data at the early production period. Based on the matching results of type curves, the well distance is slightly bigger than $500 \mathrm{~m}$.

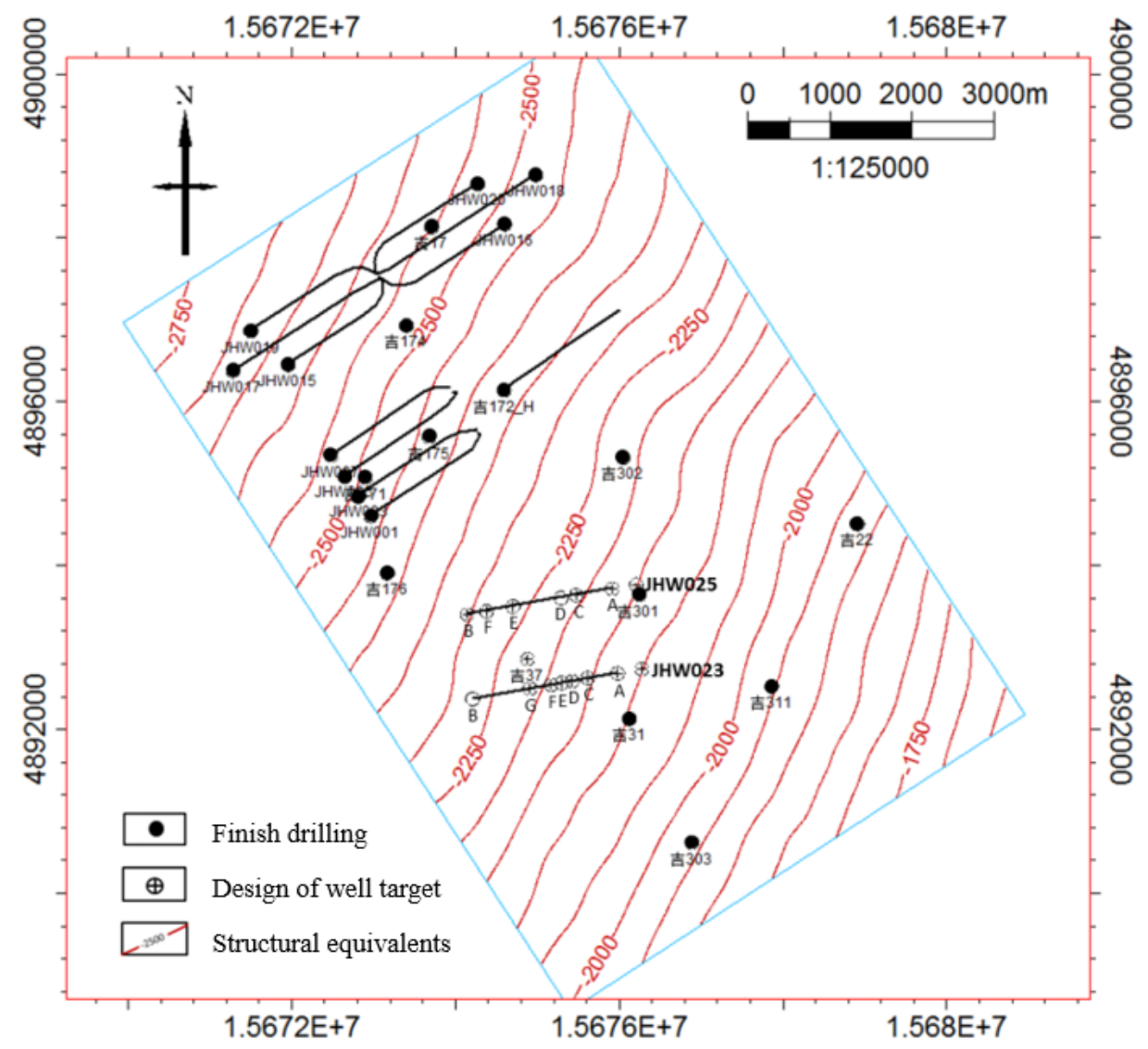

Figure 14. The scheme of the well placement in the oil field. 
Table 2. Some fracturing parameters for the well-industry-production-scheme.

\begin{tabular}{|c|c|c|c|c|c|c|}
\hline Well Names & JHW015 & JHW016 & JHW017 & JHW018 & JHW019 & JHW020 \\
\hline Well length, m & 1310 & 1312 & 1801 & 1732 & 1228 & 1304 \\
\hline Fracture stages & 18 & 18 & 23 & 23 & 15 & 17 \\
\hline Half length, m & 140 & 175 & 125 & 175 & 145 & 155 \\
\hline Porosity, \% & \multicolumn{6}{|c|}{10.99} \\
\hline Permeability, mD & \multicolumn{6}{|c|}{0.012} \\
\hline Oil saturation, $\%$ & \multicolumn{6}{|c|}{65} \\
\hline
\end{tabular}

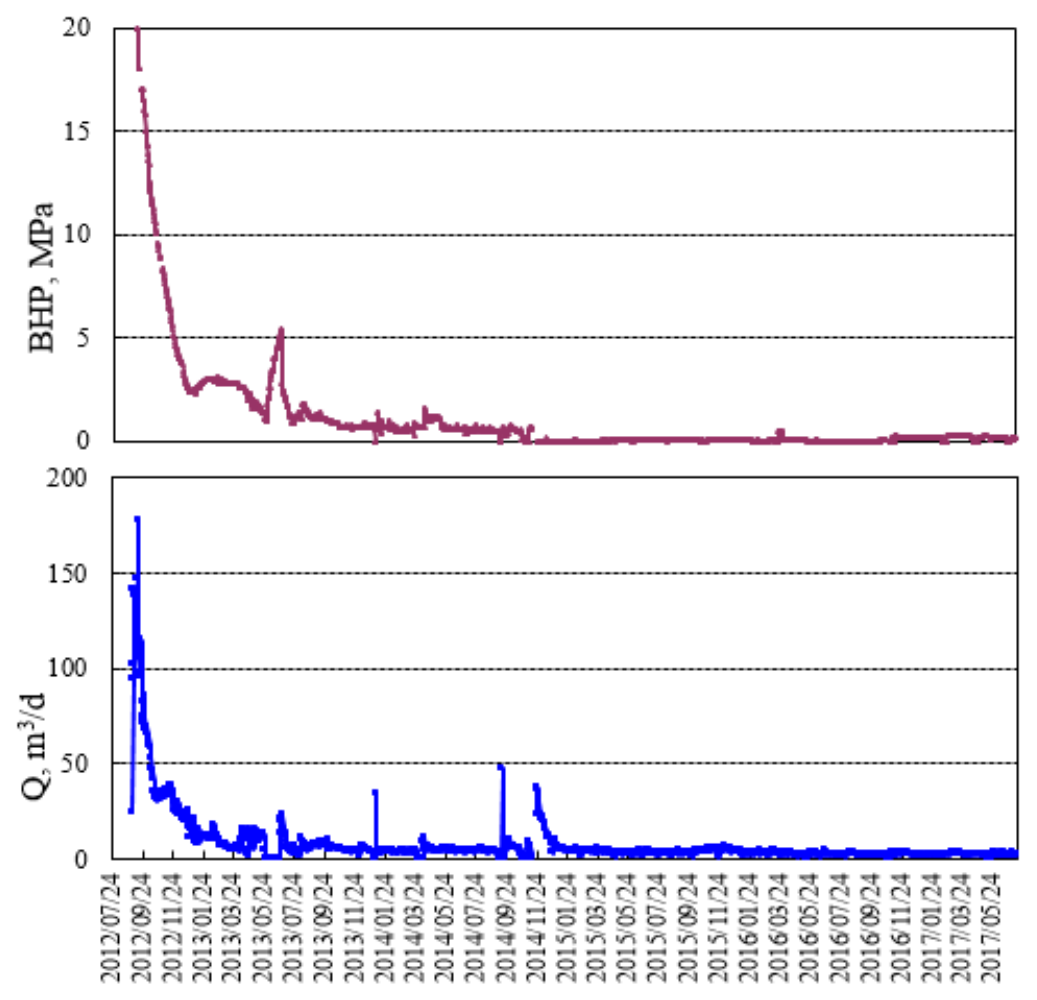

Figure 15. The scheme of the production for well JHW015.

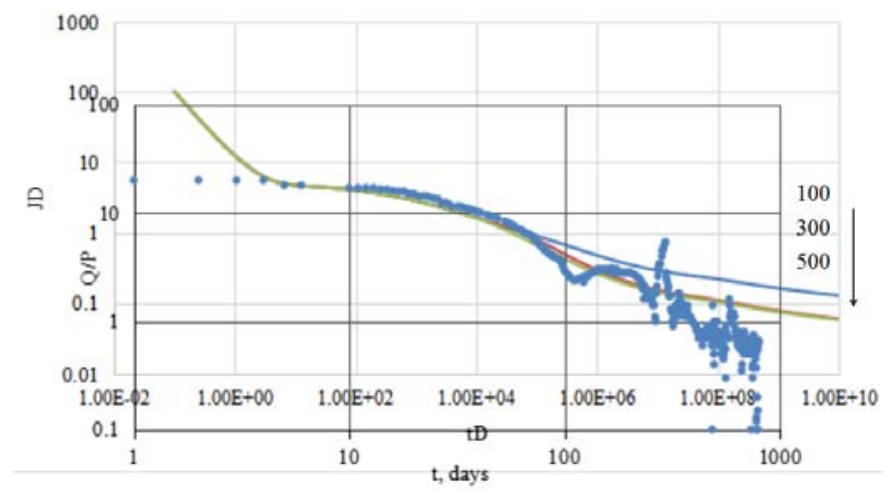

Figure 16. The results of type curves matching between data and proposed method. 


\section{Conclusions}

To gain better understanding of well performance of WIPS scheme, in this paper, a semi-analytical multi-well productivity index (MPI) model in Laplace domain is developed through considering the influences of MWPI. Through model validation and sensitivity analysis, some meaningful conclusions are summarized as follows:

- Avoiding complicated grid refinement, our proposed semi-analytical model provides promising calculation speed over numerical simulation, especially for complex fracture geometry.

- Compared to single multi-fractured horizontal wells (SMFHWs), our proposed multi-well pressure interference model has the abilities to identify the flow regimes with MWPI. Our results show that part of flow regimes are distorted by MWPI to some degree. The slope of type curves which characterize the linear or bi-linear flow regime is no longer equal to 0.5 or 0.25 . The horizontal line which characterize radial flow regime is no longer equal to 0.5 .

- Well rate mainly determines the distortion degree of MPI curves. Well rate will distort pressure and MPI curves when MWPI occurs. As the well rate decreases, the distortion of MPI curves will become severe.

- Fracture length, well spacing, fracture spacing mainly determine when the MWPI occurs. As the well spacing increases, fracture length decreases, fracture spacing decreases, the occurrence of MWPI becomes later. For well spacing, fracture spacing, when MWPI occurs, pressure curves splits, and then overlaps again. For fracture length, pressure curves will always split until MWPI reaches certain degree.

- Stress sensitivity coefficient mainly affects the MPI at the formation pseudo-radial flow stage, almost has no influence on the occurrence of MWPI. The bigger the stress sensitivity coefficient, the smaller the multi-well productivity index.

- Our application also shows promising aspects of our semi-analytical model to estimate the well distance between multi-wells for MWPP scheme, which is often uncertain in the hydraulic fracturing operation.

Author Contributions: Guangfeng Liu and Zhan Meng designed the research; Lu Wang and Yan Cui builded the model; Shenglai Yang and Chenggang Liang analyzed CMG experimental data; Zhan Meng and Guangfeng Liu in charge of writing.

Acknowledgments: The authors gratefully acknowledge the financial support from National Natural Science Foundation of China (Grant No. 51574257, Grant No. 51404282) and National 973 Project (2015CB250900).

Conflicts of Interest: The authors declare no conflict of interest.

\section{Abbreviations}

$\begin{array}{ll}\text { WIPS } & \text { well-industry-production-scheme } \\ \text { MWPI } & \text { multi-well pressure interference } \\ \text { single multi-fractured horizontal wells } \\ \text { SMFHWs } & \text { multiwell productivity index } \\ \text { MPI } & \text { natural fractures } \\ \text { NFs } & \text { hydraulic fractures } \\ \text { HFs } & \text { Stimulated Reservoir Volume } \\ \text { SRV } & \text { dimensionless pressure } \\ \text { DP } & \text { dimensionless pressure derivation } \\ \text { DPD } & \end{array}$




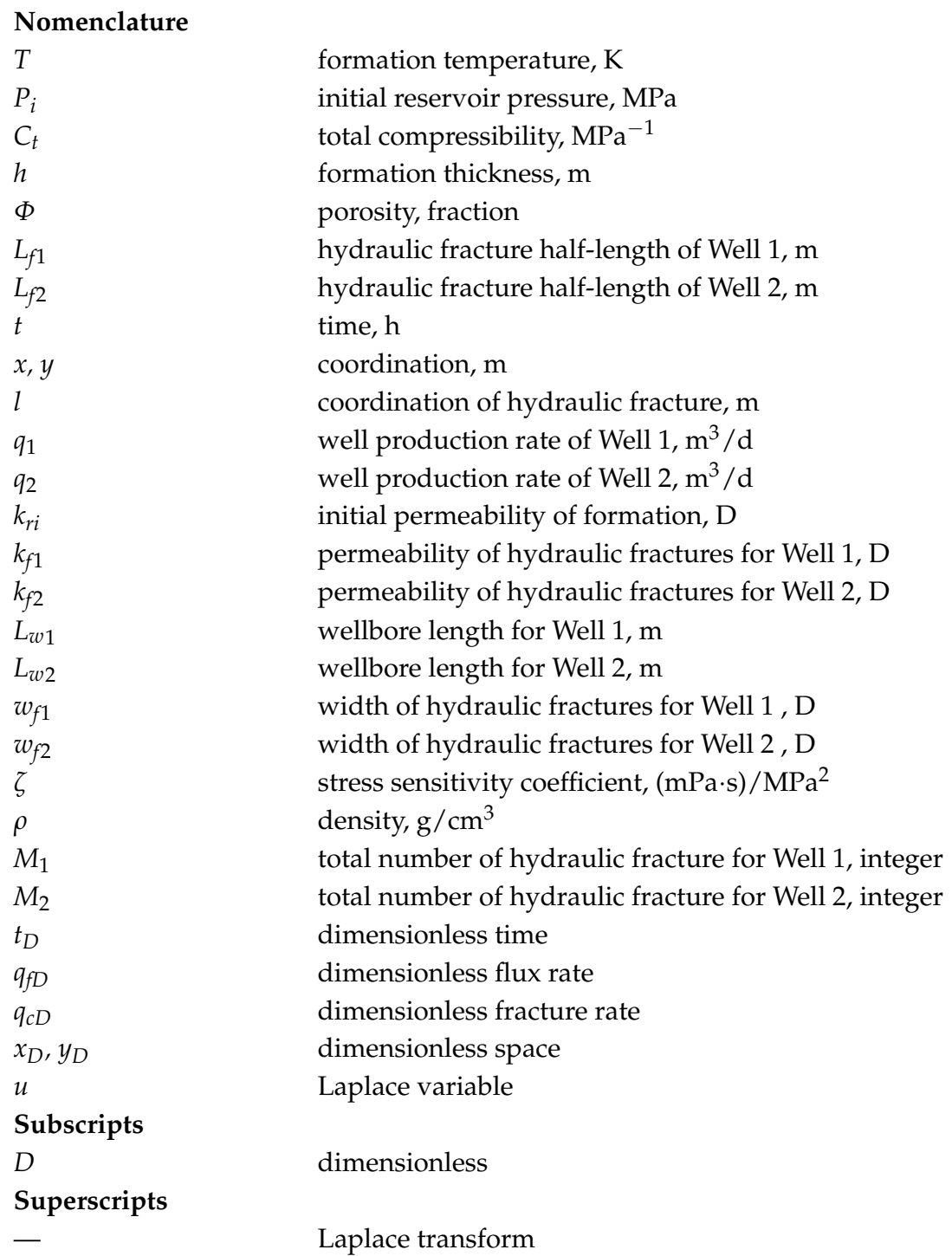

\section{References}

1. Tight Oil Expected to Make up Most of U.S. Oil Production Increase Through 2040. Available online: https: / / www.eia.gov / todayinenergy/ detail.php?id=29932\# (accessed on 22 February 2017).

2. Hu, J.; Zhang, C.; Rui, Z.; Yu, Y.; Chen, Z. Fractured horizontal well productivity prediction in tight oil reservoirs. J. Pet. Sci. Eng. 2017, 151, 159-168. [CrossRef]

3. Ozkan, E.; Raghavan, R. New solutions for well-test analysis problems. Part 1. Analytical considerations. SPE Form. Eval. 1991, 6, 359-368. [CrossRef]

4. Ozkan, E.; Raghavan, R. New Solutions for Well-Test-Analysis Problems: Part 2. Computational Considerations and Applications. SPE Form. Eval. 1991, 6, 369-378. [CrossRef]

5. Guo, G.; Evans, R.D. Pressure-Transient Behavior and Inflow Performance of Horizontal Wells Intersecting Discrete Fractures. In Proceedings of the SPE Annual Technical Conference and Exhibition, Houston, TX, USA, 3-6 October 1993. SPE 26446.

6. Wan, J.; Aziz, K. Semi-Analytical Well Model of Horizontal Wells with Multiple Hydraulic Fractures. SPE J. 2002, 7, 437-445. [CrossRef]

7. Al-Kobaisi, M.; Ozkan, E.; Kazemi, H. A Hybrid Numerical-Analytical Model of Finite-Conductivity Vertical Fractures Intercepted by a Horizontal Well. In Proceedings of the SPE International Petroleum Conference in Mexico, Puebla, Mexico, 7-9 November 2004. SPE 92040. 
8. Mayerhofer, M.; Lolon, E.; Youngblood, J.; Heinze, J. Integration of Microseismic Fracture Mapping Results with Numerical Fracture Network Production Modeling in the Barnett Shale. In Proceedings of the SPE Annual Technical Conference and Exhibition, San Antonio, TX, USA, 24-27 September 2006. SPE 102103.

9. Ozkan, E.; Brown, M.; Raghavan, R.; Kazemi, H. Comparison of Fractured Horizontal-Well Performance in Conventional and Unconventional Reservoirs. Dermatol. Surg. 2009, 27, 703-708.

10. Ozkan, E.; Brown, M.L.; Raghavan, R.; Kazemi, H. Comparison of Fractured-Horizontal-Well Performance in Tight Sand and Shale Reservoirs. SPE Reserv. Eval. Eng. 2011, 14, 248-259. [CrossRef]

11. Brown, M.L.; Ozkan, E.; Raghavan, R.S.; Kazemi, H. Practical Solutions for Pressure Transient Responses of Fractured Horizontal Wells in Unconventional Reservoirs. J. Pet. Technol. 2010, 62, 63-64.

12. Brown, M.L.; Ozkan, E.; Raghavan, R.S.; Kazemi, H. Practical Solutions for Pressure-Transient Responses of Fractured Horizontal Wells in Unconventional Shale Reservoirs. SPE Reserv. Eval. Eng. 2011, 14, $663-676$. [CrossRef]

13. Stalgorova, E.; Mattar, L. Practical Analytical Model to Simulate Production of Horizontal Wells with Branch Fractures. In Proceedings of the SPE Canadian Unconventional Resources Conference, Calgary, AB, Canada, 30 October-1 November 2012. SPE 162515.

14. Stalgorova, E.; Mattar, L. Analytical Model for History Matching and Forecasting Production in Multifrac Composite Systems. In Proceedings of the SPE Canadian Unconventional Resources Conference, Calgary, AB, Canada, 30 October-1 November 2012. SPE 162516.

15. Wang, L.; Wang, X.; Li, J.; Wang, J. Simulation of Pressure Transient Behavior for Asymmetrically Finite-Conductivity Fractured Wells in Coal Reservoirs. Transp. Porous Media 2013, 97, 353-372. [CrossRef]

16. Deng, J.; Zhu, W.; Ma, Q. A new seepage model for shale oil reservoir and productivity analysis of fractured well. Fuel 2014, 124, 232-240. [CrossRef]

17. Wang, W.; Shahvali, M.; Su, Y. A semi-analytical fractal model for production from tight oil reservoirs with hydraulically fractured horizontal wells. Fuel 2015, 158, 612-618. [CrossRef]

18. Fan, D.; Yao, J.; Hai, S.; Hui, Z.; Wei, W. A composite model of hydraulic fractured horizontal well with stimulated reservoir volume in tight oil \& oil reservoir. J. Nat. Oil Sci. Eng. 2015, 24, 115-123.

19. Xiao, C.; Tian, L.; Yang, Y.; Zhang, Y.; Gu, D.; Chen, S. Comprehensive application of semi-analytical PTA and RTA to quantitatively determine abandonment pressure for $\mathrm{CO}_{2}$ storage in depleted shale oil reservoirs. J. Pet. Sci. Eng. 2016, 146, 813-831. [CrossRef]

20. Sardinha, C.M.; Petr, C.; Lehmann, J.; Pyecroft, J.F. Determining Interwell Connectivity and Reservoir Complexity through Frac Pressure Hits and Production Interference Analysis. In Proceedings of the SPE/CSUR Unconventional Resources Conference-Canada, October, Calgary, AB, Canada, 30 September-2 October 2014. SPE 171628.

21. Awada, A.; Santo, M.; Lougheed, D.; Xu, D.; Virues, C. Is That Interference? A Work Flow for Identifying and Analyzing Communication through Hydraulic Fractures in a Multiwell Pad. SPE J. 2016, 21, 1-554. [CrossRef]

22. Jia, P.; Cheng, L.; Huang, S.; Cao, R.; Xu, Z. A Semi-Analytical Model for Production Simulation of Complex Fracture Network in Unconventional Reservoirs. In Proceedings of the SPE/IATMI Asia Pacific Oil \& Oil Conference and Exhibition, Nusa Dua, Bali, Indonesia, 20-22 October 2015. SPE 176227.

23. Zeng, F.B.; Zhao, G.; Liu, H. A New Model for Reservoirs with a Discrete-Fracture System. J. Can. Pet. Technol. 2012, 51, 127-136. [CrossRef]

24. Chen, Z.; Liao, X.; Zhao, X.; Lv, S.; Zhu, L. A Semianalytical Approach for Obtaining Type Curves of Multiple-Fractured Horizontal Wells with Secondary-Fracture Networks. SPE J. 2016, 21, 538-549. [CrossRef]

25. Zhou, W.; Banerjee, R.; Poe, B.; Spath, J.; Thambynayagam, M. Semianalytical Production Simulation of Complex Hydraulic-Fracture Networks. SPE J. 2013, 19, 6-18. [CrossRef]

26. Stehfest, H. Algorithm 368: Numerical inversion of Laplace transforms [D5]. Commun. ACM 1970, 13, 47-49. [CrossRef]

(C) 2018 by the authors. Licensee MDPI, Basel, Switzerland. This article is an open access article distributed under the terms and conditions of the Creative Commons Attribution (CC BY) license (http:/ / creativecommons.org/licenses/by/4.0/). 